\title{
BMJ Open Quality Improving care collaboration for NICU patients to decrease length of stay and readmission rate
}

\author{
Cherrie D Welch, ${ }^{1}$ Jennifer Check, ${ }^{1}$ T Michael O'Shea ${ }^{2}$
}

To cite: Welch CD, Check J, O'Shea TM. Improving care collaboration for NICU patients to decrease length of stay and readmission rate.BMJ Open Quality 2017;6:e000130. doi:10.1136/ bmjoq-2017-000130

- Additional material is published online only. To view please visit the journal online (http://dx.doi.org/10.1136/ bmjoq-2017-000130).

Received 30 May 2017 Accepted 15 August 2017

CrossMark

${ }^{1}$ Division of Neonatology, Department of Pediatrics, Wake Forest Baptist Health, Wake Forest School of Medicine, Winston-Salem, North Carolina, USA

2Division of Neonatology, Department of Pediatrics, UNC Hospitals, University of North Carolina at Chapel Hill, Chapel Hill, North Carolina, USA

Correspondence to Dr Jennifer Check; jcheck@wakehealth.edu

\begin{abstract}
Background Medically complex patients in neonatal intensive care units (NICUs) typically require long hospitalisations and care from multiple subspecialists. Scheduled multidisciplinary discussions could improve collaboration and continuity of care and thereby improve patient outcomes. The specific aims of the project were to decrease the average length of hospitalisation by at least 1 day and improve parent satisfaction ratings on a standard questionnaire by the end of our project's first year, and to maintain a stable (or decreased) cause-related (30-day) readmission rate.
\end{abstract}

Methods We designed a quality improvement project to enhance collaboration and continuity of care for medically complex infants cared for in the NICU of Brenner Children's Hospital. Weekly multidisciplinary team meetings were held to discuss the long-term plan for patients who met specific criteria. Attendees included attending neonatologists, paediatric surgeons, a physical therapist, an occupational therapist, a speech therapist, a social worker, a nurse coordinator for palliative care, a family support coordinator, the NICU Nurse Manager, a hospital chaplain, mid-level providers, bedside nurses, a nurse quality improvement leader and the leaders and database manager for the quality improvement project. When needed for specific patients, a bioethicist was included. Results One year after implementing the project, the average duration of hospitalisation had decreased by 6.5 days. Cause-related readmission rates decreased from $3.33 \%$ to $0.95 \%$. Parent satisfaction scores did not change significantly.

Conclusions Weekly multidisciplinary meetings to coordinate and provide continuity of care for medically complex neonates in our NICU was associated with improved patient outcomes.

\section{INTRODUCTION}

Many infants admitted to a neonatal intensive care unit (NICU) have complex medical conditions requiring care from multiple subspecialists and prolonged hospitalisations. In our NICU at Brenner Children's Hospital, continuity of care for infants with extended hospitalisations is challenging because attending neonatologists rotate every 1 to 2 weeks. Also, coordination of care among subspecialists is difficult because of competing demands for time. Diminished collaboration and continuity of care can impact negatively on length of hospitalisation, ${ }^{1}$ patient outcomes ${ }^{2}$ and parents' and healthcare providers' well-being. ${ }^{3}$

The Care Collaboration for Babies with Extended Stays (CBES) project was designed as a quality improvement intervention to increase continuity and collaboration of care for infants hospitalised in the NICU at Brenner Children's Hospital who were expected to have prolonged hospitalisations. The intervention consisted of weekly multidisciplinary discussions about a selected subset of patients, and each discussion concluded with a set of recommendations that were communicated to the rest of the patient's care team and documented in the patient's medical record.

During and following implementation, data were collected on length of hospitalisation, rehospitalisations within 30 days of discharge from the NICU, parents' satisfaction and physician's evaluation of the impact of the intervention on physician autonomy, as well as process variables, including duration of the weekly meetings and number of disciplines involved.

\section{METHODS}

Project goals

The first goal was to decrease average length of hospitalisation by at least 1 day. The second goal was to increase overall parent satisfaction on Press Ganey to 95\% (baseline 93.8\%) as well as improve ratings on the following HowsYourBabyNC.org survey questions: (1) How often have you been able to talk with the same doctors?-increase by $20 \%$ (from $56 \%$ to $67 \%$ ) in those responding 'just right'; (2) How often have you been able to participate in decision making about your baby's care?-increase by $10 \%$ (from $76 \%$ to $83 \%$ ) in those responding 'just right'; (3) If your infant was discharged with home equipment, how comfortable were you with operating the equipment?-increase by $20 \%$ (from $67 \%$ to $80 \%$ ) in those responding 'comfortable'. 
Table 1 Inclusion criteria

Grade 4 intraventricular hemorrhage and/or anticipated need for ventriculoperitoneal shunt.

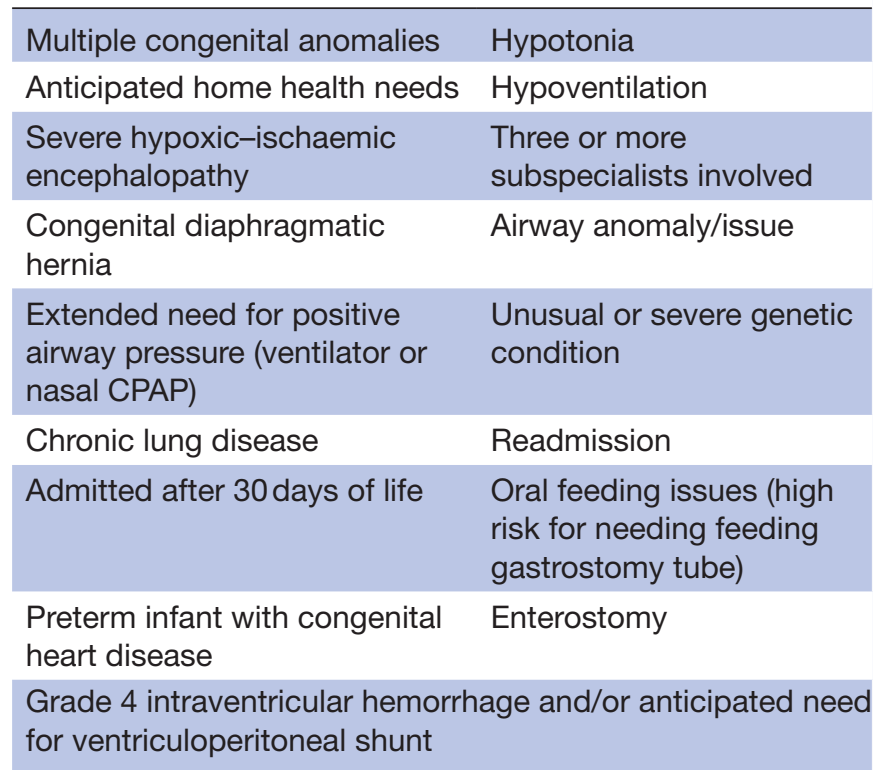

CPAP, continuous positive airway pressure.

Additional goals were to maintain a stable (or decreased) related cause (30-day) readmission rate for NICU discharges by the end of the first year and to have physician autonomy and physician-patient relationship not impacted more than 'very little' as assessed by questionnaire.

\section{Patients eligible for the project}

Patients were considered for inclusion in this quality improvement project if they met one or more of the criteria listed in table 1 , either on admission or during their hospital course. ${ }^{45}$

\section{Planning the intervention and evaluation}

The intervention and primary outcomes for this project were developed initially in consultation with the medical director of the NICU, an experienced project leader of quality improvement projects. A preliminary proposal was then presented to a large group of stakeholders, including nurses, a social worker and a parent advocate, and changes suggested by stakeholders were incorporated prior to initiation of the project.

After the intervention began, the project leaders met monthly to discuss interim results and plan and implement modifications to the design which were tested via Plan-Do-Study-Act (PDSA) cycles. ${ }^{6}$

\section{Intervention}

The primary component of the intervention is a weekly 1-hour multidisciplinary meeting of health professionals at which medically complex neonates are discussed throughout their hospital course with the purpose of (1) identifying and removing barriers to progression of care and discharge and (2) arranging for a smooth transition to home and posthospital care by identifying resources in the community and ensuring adequate follow-up. ${ }^{78}$

This strategy was selected because it enhances continuity of care by involving a consistent group of professionals with expertise in managing chronic illness in children, increases communication among healthcare professionals representing multiple specialties and takes a comprehensive view of the patient, with a focus on barriers and facilitators to the transition from the hospital to the home, improving efficiency and safety of care. ${ }^{910}$

This intervention is also family centred in that attention is paid explicitly to the family's understanding of their infant's medical issues, coping ability, financial resources, other barriers to engaging in care and their degree of comfort in caring for their infant postdischarge. ${ }^{11} 12$ The principles of continuous, coordinated and comprehensive care are foundational for managing chronic illness. ${ }^{13}$

We initially planned to involve parents in the meetings when their child was discussed; however, we were unable to implement this component without detracting from the efficiency of the process. Instead, we implemented a process whereby details of the discussion would be communicated to the parents within 24 hours of the meeting and their questions and input brought back to the group the following week.

The other major challenge that we anticipated was that decisions made by the multidisciplinary group could be in conflict with management plans made by attending neonatologists. We planned to address this issue by sharing our interim results with all involved neonatologists and paediatric surgeons so that they could see the benefit to patients resulting from more consistent, coordinated, comprehensive care. As a balance measure, we surveyed the attending neonatologists regarding their perceptions about whether the project was interfering with physician autonomy and/or the physician-patient relationship.

While we anticipated that this initiative would reduce the overall length of hospitalisation for these infants, we wanted to ensure that a concomitant increase in readmission rates did not occur. Thus, we followed each of the patients for 1 month after discharge to determine whether or not they had been readmitted.

Lastly, we anticipated that for many of the babies served by the project, there would be a high degree of uncertainty about the quality of life and probability for survival, resulting in ethical issues. Thus, hospital ethicists were involved in the discussions on an 'as needed' basis.

\section{Setting}

Brenner Children's Hospital Intensive Care Nursery (ICN) is a 38-bed level 4 unit that receives referrals from a large geographical area in Northwest North Carolina, Eastern Tennessee and Southwest Virginia. Nearly all patients are born at other hospitals and are transported to Brenner. All major paediatric subspecialties are available at Brenner, including all paediatric surgical subspecialties except transplantation surgery. 


\section{Specifics}

On the morning of the meeting, one of three neonatologists involved in this initiative would review the electronic medical record of each infant in the Brenner nursery and identify newly admitted patients who were eligible for inclusion in this process and to gain updated information about the patients already being served by the process. A table listing each patient and their major diagnoses, barriers to discharge and goals was updated each week (online supplementary appendix A). The neonatologist organising the meeting would select up to six infants, out of those on the list, for discussion, with priority given to those infants for whom the multidisciplinary discussion was deemed likely to be particularly helpful in progressing the care of the patient beyond its current stage. The pertinent details of the discussion were documented in the medical record using a template designed for this purpose.

\section{Evaluation of the project \\ Process measures}

Each week we recorded whether at least five of the following six key disciplines were present at the meeting: neonatologist, paediatric surgeon or surgery nurse practitioner, social worker, palliative care nurse specialist, ICN nurse specialist and an occupational, speech or physical therapist. We also recorded the duration of each meeting. For each month, we recorded the per cent of eligible patients who were discussed.

\section{Outcome measures}

To evaluate the impact on average length of hospitalisation, we obtained data from the hospital database for length of stay for NICU patients overall in the year preceding the implementation of this project and compared it with the length of stay for all patients during the first year of the project. Family satisfaction with care was assessed using parent responses to the Press Ganey survey and the HowsYourBabyNC.org survey.

\section{Balance measures}

Using Survey Monkey, we surveyed neonatologists, approximately 6 and 12 months after initiation of the project, about their perception of how much the project interfered with physician autonomy. Data about readmission after discharge from the NICU were collected by reviewing each patient's medical record 1 month after discharge to ascertain whether the infant had been readmitted to our hospital. We did not collect information about hospitalisations at other hospitals and assumed that patients with complex medical conditions would have been referred to our hospital if readmission was required.

\section{RESULTS}

\section{Characteristics of infants}

During the first year, 71 infants were included in this initiative. The average birth weight (SD) was 1663 (1174) g. The average (SD) gestational age at birth was $303 / 7$

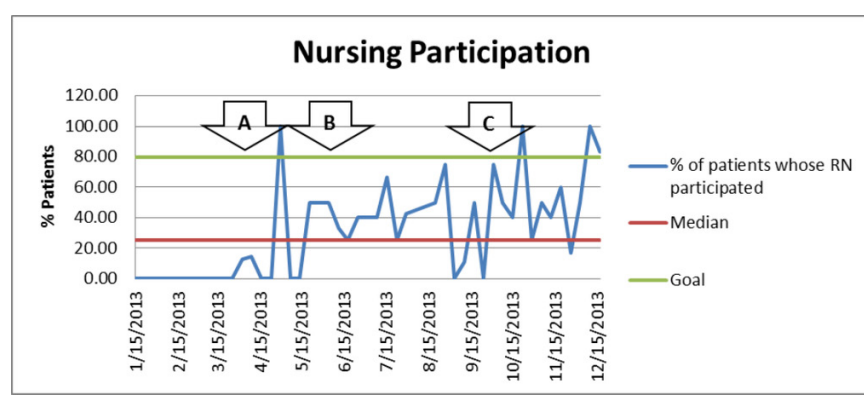

Figure 1 Run chart of percentage of patient discussions, by week, in which the bedside nurse participated. Arrow A: change in time of meeting. Arrow B: education of nurses regarding process. Arrow $\mathrm{C}$ : day of meeting notification of patients to be discussed.

(6.2) weeks. The three most common eligibility criteria were chronic lung disease, oral feeding difficulties and having three or more subspecialty services involved. Twenty-nine patients $(40 \%)$ were discharged home with medical equipment (18 with a gastrostomy tube, 12 with a cardiorespiratory monitor, 12 with supplemental oxygen, 2 with mechanical ventilation).

\section{Process measures}

The average (SD) duration of weekly meetings was 58.92 (4.51) min. Twenty per cent of meetings exceeded $60 \mathrm{~min}$.

In $83.7 \%$ of meetings, at least five of the following six key disciplines were present. Due to their unpredictable schedules, a representative from the paediatric surgery service was present at only $60 \%$ of the meetings. Infants with whom the paediatric surgery service was involved were discussed first to accommodate the surgeons' availability and facilitate their participation.

The bedside nurse was initially not listed among the 'Key Disciplines'; however, it was quickly recognised that the insights and input from the bedside nurse are extremely helpful in discussions involving feeding issues and family engagement, understanding and coping. Much effort was put towards involving and engaging the bedside nurse in this process. Initially, they were simply encouraged to participate in the discussions. However, the timing of the meeting had inadvertently been scheduled at the time of nursing shift change. The time was adjusted to facilitate nursing participation (arrow A in figure 1). However, this did not result in a significant increase in nursing participation. It was determined that the nursing staff was not fully aware of the purpose of the meeting, the importance of their input in the discussions or whether or not one of their patients was to be discussed. In order to address these barriers, the unit nurse educator provided information and began to send the weekly list of CBES patients to the nurses by email (arrow B). An additional barrier to nursing participation was identified as being difficulty in the ability of the nurses to leave the bedside to attend the meeting in the NICU conference room. The nursing leadership developed a strategy whereby the nurses would be notified an hour prior to the meeting that their patient was going to be discussed and asked to try to arrange 


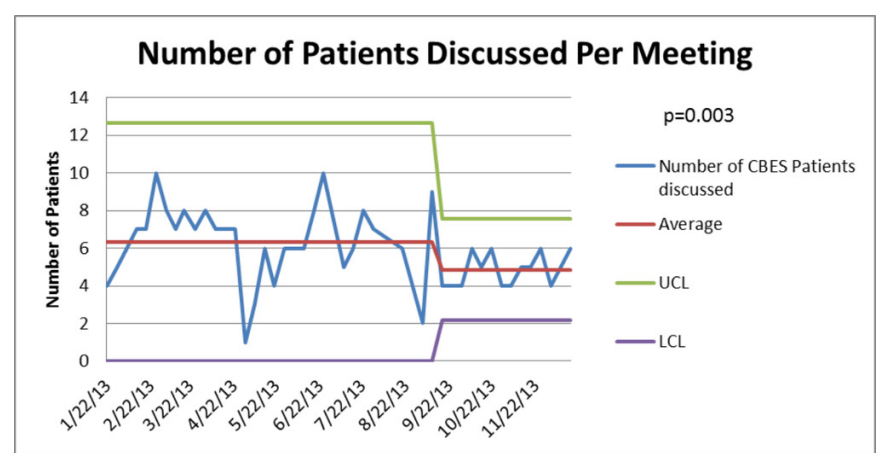

Figure 2 Control chart of the number of patients discussed per meeting. CBES, Care Collaboration for Babies with Extended Stays.

workflow/breaks in order to be available when called to come to the conference room (arrow $\mathrm{C}$ ). Despite our best efforts, it remains difficult to achieve nursing participation given the unpredictability of nursing workflow in the NICU.

On average, 14 patients were eligible for inclusion in CBES discussions each week. Initially, we chose four patients and planned to discuss them each week until discharge, only adding new patients to the process as discharges occurred. However, we soon realised that this would only serve a small proportion of infants who were likely to benefit from this service, and therefore we began to prioritise all eligible patients according to the likelihood the multidisciplinary discussion would be particularly helpful in progression of care of the patient beyond its current stage.

There was wide variation in the number of infants discussed each week during the first months of the project. However, by the end of the year, the team discussions became more consistent in level of detail and length, allowing for an effective discussion to be held on five to six patients per meeting (figure 2). The standardisation of the discussions was aided by a template of questions that was used for each new patient and then updated with each subsequent discussion (online supplementary appendix B). This template was also used in documenting the discussions in the medical record. The percentage of

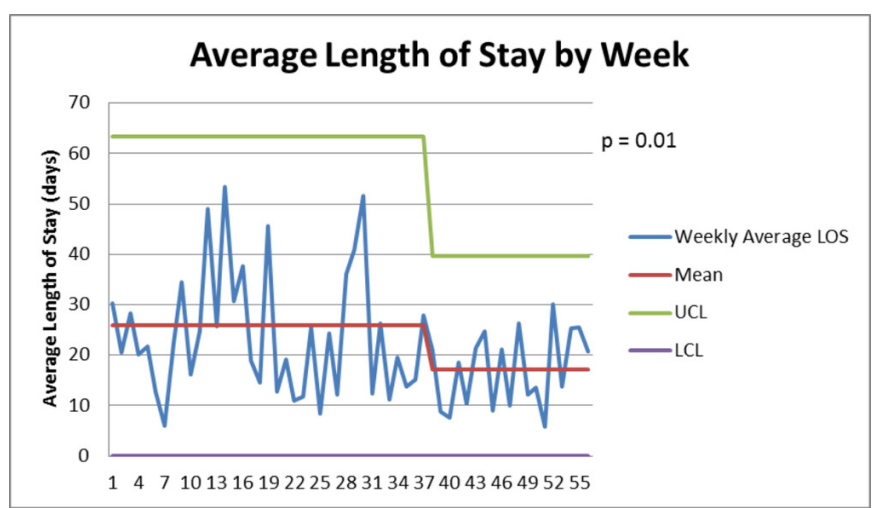

Figure 3 Control chart of length of hospital stay by week for the first year of the initiative. LOS, length of stay. eligible patients who were discussed in at least one out of every four meetings was $76.9 \%$.

\section{Outcome measures}

For the year prior to the intervention, the average length of hospitalisation for all Brenner neonatal intensive care patient discharges was 28.2 days. For the first year of the project, the average length of hospitalisation was 21.7 days. The variability in the length of hospitalisation decreased in the latter half of the first year of the initiative (figure 3).

Only seven Press Ganey surveys were returned during the year, so we did not analyse these data. Parent responses on the HowsYour BabyNC.org survey are shown in table 2.

\section{Balance measures}

The percent of neonatologists who responded that the project interfered with physician autonomy 'none' or 'very little' was $90 \%$ at 6 months after implementation and $79 \%$ after 12 months. The per cent of neonatologists who responded that the project interfered with physician-patient relationship 'very little' was $100 \%$ at 6 months after implementation and $93 \%$ after 12 months.

Of the infants who were discussed in the meetings, five (7\%) were readmitted within 30 days and two died after discharge. All readmissions were for respiratory-related disorders. Related cause readmission rates at 30 days postdischarge for all infants cared for in the Brenner ICN in the year before and after implementation of the project were $3.33 \%$ and $0.95 \%$, respectively.

\section{DISCUSSION}

There were five goals set prior to implementation of this quality improvement study. Three of the five were attained at the 1-year mark after the intervention was implemented.

Since implementation, length of stay has decreased by 6.5 days, exceeding our goal of 1 day. In addition, the intervention was associated with a reduction in readmission rates within 30 days of discharge. Both of these outcome measures directly impact the health and wellbeing of the child, as well as the well-being of the family. The continuity and collaborative care allows the team to clearly define goals for discharge, ensures the entire medical team understands the goals set forth and streamlines the discharge planning by giving the family a more efficient and effective process. The third goal attained was that there was no significant impact on physician autonomy. This goal helps contribute to the physician and parent satisfaction level as well as protecting the integrity between the physician-patient relationship.

The two goals for which we did not see effective change were increased parent satisfaction on Press Ganey surveys and improved ratings on HowsYourBabyNC.org. These findings were multifactorial. First, there were only seven Press Ganey surveys returned throughout the year. Also, the decision to not have parents attend the multidisciplinary meetings may have contributed to this lack of 


\begin{tabular}{llll}
\hline Question & $\begin{array}{l}\text { Baseline \%, Postimplementation \%, } \\
\mathbf{n = 3 7 2}\end{array}$ & $\begin{array}{l}\text { n=350 } \\
\text { p Value }\end{array}$ \\
\hline $\begin{array}{l}\text { How often have you been able to talk with the same doctors? ('just } \\
\text { right') }\end{array}$ & 56 & 72 \\
$\begin{array}{l}\text { How often have you been able to participate in decision making } \\
\text { about your baby's care? ('just right') }\end{array}$ & 76 & 72 \\
$\begin{array}{l}\text { If your infant is discharged with home equipment, how comfortable } \\
\text { were you with operating the equipment? ('very comfortable') }\end{array}$ & 67 & 67 & 0.21 \\
\hline
\end{tabular}

change. There was much debate regarding parent attendance at the weekly meeting; however, in order to maintain efficiency in each patient discussion with such a large group, the decision remained to not have parents attend. Throughout this year, we have encouraged the primary medical team to relay key points of our discussion to the family as well as bring back questions for the multidisciplinary team. This process of informing the parents has not been standardised and may have impacted the parents' perception of participation in decision making in relation to this weekly care coordination meeting. ${ }^{14} \mathrm{We}$ also see from the questionnaire that additional efforts are needed to increase the family's level of comfort with operating home equipment.

Another area for improvement is attendance by bedside nursing. ${ }^{15}$ Three interventions were undertaken to improve nursing participation (figure 1). After the third intervention, that of notifying the appropriate nursing staff 1 hour prior to the meeting which patients were to be discussed, participation improved.

This quality improvement endeavour can be generalisable to other level 3 and 4 NICUs as these are the units likely to have patients with chronic, complex medical and surgical illnesses requiring multiple subspecialists. As these types of patients are most at-risk for prolonged hospitalisations and readmissions, this population is likely to receive the most benefit from this type of coordinated care. ${ }^{16}$ Our intervention focuses on two key goals: (1) identify and remove barriers to progression of care and ultimately discharge and (2) arrange for a smooth transition to home and posthospital care by identifying resources in the community and ensuring adequate follow-up. The engagement of the wide range of disciplines involved in all facets of the care of these infants is essential in the success of this type of strategy.

In order to monitor and maintain improvement, quarterly meetings were arranged to review progress, address obstacles and conduct PDSA cycles on various measures. As we see in figure 3, average length of stay was reduced and the variability in length of hospitalisation decreased in the latter half of the first year. Also, related cause readmission rates at 30 days postdischarge were overall decreased. Conducting periodic physician surveys and monitoring parent feedback from HowsYourBabyNC.org will allow us to continue to evaluate the progress of this intervention.
The only 'cost' of this intervention is the time invested by each member of the team. An attending neonatologist takes time to prepare for the meeting by reviewing all neonatal patients and determines which meet criteria for discussion. Each member gives 1 hour of their time each week for the meeting. Due to the variability in each patient's and family's need, the number of hours outside of the meeting dedicated to coordination of care are unable to be tallied. On the other hand, the decreased length of stay and decreased readmission rates are evidence of cost-saving measures to the family and healthcare system.

The innovative aspect of this initiative compared with other multidisciplinary discharge meetings is that patients were identified on admission as being at high risk for extended hospitalisation and were subsequently followed and discussed by the team throughout their hospital stay, not just when discharge became imminent. This allowed the group to proactively and effectively identify and address barriers to progression of care throughout the hospital course in addition to focusing on the ultimate discharge. Also, many multidisciplinary meetings lose effectiveness over time as they attempt to cover too many patients at each meeting and thus the meetings are long and attrition of key members is high. By prioritising six infants out of the entire NICU census for discussion each week, the meeting is able to be kept to $60 \mathrm{~min}$, with an appropriate in-depth discussion of each patient.

In conclusion, this quality improvement intervention has proven to be effective in reducing length of stay and readmission rates through collaboration and coordination of care for our most complex infants. With further improvement in family and staff involvement, the expectation is that collaboration will continue to improve and will be evidenced in satisfaction scores and patient outcomes.

Contributors CDW conceptualised and designed this quality improvement study, collected the data, analysed and interpreted the data, drafted the initial manuscript and approved the final manuscript as submitted. JC helped collect the data, assisted in analysis of the data, helped draft and revise the manuscript and approved the final manuscript as submitted. TMOS helped conceptualise the study, assisted in analysis of the data, helped draft and revise the manuscript and approved the final manuscript as submitted.

Competing interests None declared.

Provenance and peer review Not commissioned; internally peer reviewed.

Open Access This is an Open Access article distributed in accordance with the Creative Commons Attribution Non Commercial (CC BY-NC 4.0) license, which 
permits others to distribute, remix, adapt, build upon this work non-commercially, and license their derivative works on different terms, provided the original work is properly cited and the use is non-commercial. See: http://creativecommons.org/ licenses/by-nc/4.0/

(c) Published by the BMJ Publishing Group Limited. For permission to use (where not already granted under a licence) please go to http://www.bmj.com/company/ products-services/rights-and-licensing/

\section{REFERENCES}

1. Blecker S, Shine D, Park N, et al. Association of weekend continuity of care with hospital length of stay. Int J Qual Health Care 2014;26:530-7.

2. Zwarenstein M, Goldman J, Reeves S. Interprofessional collaboration: effects of practice-based interventions on professional practice and healthcare outcomes. Cochrane Database Syst Rev 2009:CD000072.

3. Boos VD, Okah FA, Swinton $\mathrm{CH}$, et al. The comprehensive care rounds: facilitating multidisciplinary communication among caregivers of complex patients in the neonatal intensive care unit. Adv Neonatal Care 2010;10:301-6.

4. Burns KH, Casey PH, Lyle RE, et al. Increasing prevalence of medically complex children in US hospitals. Pediatrics 2010;126:638-46.

5. Berry JG, Agrawal R, Kuo DZ, et al. Characteristics of hospitalizations for patients who use a structured clinical care program for children with medical complexity. J Pediatr 2011;159:284-90.

6. Horbar JD, Plsek PE, Leahy K. NIC/Q 2000. NIC/Q 2000: establishing habits for improvement in neonatal intensive care units. Pediatrics 2003;111:e397-410.
7. American Academy of Pediatrics, Committee on Fetus and Newborn. Hospital discharge of the high risk neonate-proposed guidelines. Pediatrics 1998;102:411-7.

8. Robison M, Pirak C, Morrell C. Multidisciplinary discharge assessment of the medically and socially high-risk infant. J Perinat Neonatal Nurs 2000;13:67-86.

9 Mills MM, Sims DC, Jacob J. Implementation and case-study results of potentially better practices to improve the discharge process in the neonatal intensive care unit. Pediatrics 2006;118(Suppl 2):S124-S133.

10 Leonard M, Graham S, Bonacum D. The human factor: the critical importance of effective teamwork and communication in providing safe care. Qual Saf Health Care 2004;13(Suppl 1):i85-i90.

11. Broedsgaard A, Wagner $L$. How to facilitate parents and their premature infant for the transition home. Int Nurs Rev 2005;52:196-203.

12. Smith VC, Hwang SS, Dukhovny D, et al. Neonatal intensive care unit discharge preparation, family readiness and infant outcomes: connecting the dots. J Perinatol 2013;33:415-21.

13. Gill A, Kuluski K, Jaakkimainen L, et al. "Where do we go from here?" Health system frustrations expressed by patients with multimorbidity, their caregivers and family physicians. Healthc Policy 2014;9:73-89.

14. Co JP, Ferris TG, Marino BL, et al. Are hospital characteristics associated with parental views of pediatric inpatient care quality? Pediatrics 2003;111:308-14.

15. Helder OK, Verweij JC, van Staa A. Transition from neonatal intensive care unit to special care nurseries: experiences of parents and nurses. Pediatr Crit Care Med 2012;13:305-11.

16. Gordon JB, Colby HH, Bartelt T, et al. A tertiary care-primary care partnership model for medically complex and fragile children and youth with special health care needs. Arch Pediatr Adolesc Med 2007;161:937-44. 